\title{
Reactive power management in photovoltaic installations connected to low-voltage grids to avoid active power curtailment.
}

\author{
J.F. Gómez-González ${ }^{1}$, D. Cañadillas-Ramallo², B. González-Díaz ${ }^{1}$, J.A. Méndez-Pérez ${ }^{3}$, J. \\ Rodríguez $^{4}$, J. Sánchez ${ }^{4}$ and R. Guerrero-Lemus ${ }^{2 *}$ \\ ${ }^{1}$ Departamento de Ingeniería Industrial, Escuela Superior de Ingeniería y Tecnología, \\ Universidad de La Laguna (ULL), Escuela Superior de Ingeniería y Tecnología, Universidad de La Laguna (ULL), \\ Apdo. 456, 38206 La Laguna (Tenerife), España. \\ ${ }^{2}$ Departamento de Física, Facultad de Ciencias, Universidad de La Laguna (ULL), \\ Apdo. 456, 38206 La Laguna (Tenerife), España, Phone +34922318306 email: rglemus@ull.edu.es. \\ ${ }^{3}$ Departamento de Ingeniería Informática y de Sistemas, Escuela Superior de Ingeniería y Tecnología, \\ Universidad de La Laguna (ULL), Apdo. 456, 38206 La Laguna (Tenerife), España. \\ ${ }^{4}$ ENDESA Distribución Eléctrica SL. C/ Ribera del Loira 60, 28042 Madrid. España.
}

\begin{abstract}
Photovoltaic (PV) inverters are traditionally designed to operate with unity power factors. In order to use reactive power capabilities of smart inverters, in this work two strategies are analysed: limiting the amount of active power delivered or oversizing the inverter. The first of these options implies a reduction in the PV production and therefore, it would lead to reduced earnings for the PV system owner. On the other hand, oversizing the PV inverter allows having reactive power compensation capabilities, while delivering full power output from its PV field.
\end{abstract}

\section{Key words}

Photovoltaic, reactive power management, electrical systems.

\section{Introduction}

Voltage related issues are the most critical among all the problems of interconnecting photovoltaic (PV) systems to the Low Voltage (LV) networks. Some of the technical solutions proposed in the literature are focussed to mitigate the variation voltage effects and achieve an effective voltage regulation throughout the network. Moreover, voltage regulation strategies can be implemented from two different point of view: from the Distribution System Operators (DSO) and from the PV systems. For that reason, the proposed solutions are divided in these two categories.

\section{Solutions provided by Distribution System Operators}

DSO are the responsible of maintaining the power quality in the LV network. Traditionally, overvoltage problem used to be solved by grid reinforcement of the LV line. However, this is an expensive measure and therefore, other solutions have been analysed and tested in order to the reduce the final operational cost. Despite of this, this solution is only contemplated as last solution to be implemented. In spite of this, some new assets are available for voltage regulation by the DSO, which can contribute to both increase the power quality and the hosting capacity of distribution grids.

On-Load Tap Changers (OLTC) are essential parts of distribution networks, and they are usually located at the substations. Their control is based on changing the transformer winding ratio by stepping up or down the taps without disconnecting the loads, causing a variation in the busbar voltage level at the substation [1]. To use them as voltage regulator devices under PV penetration scenarios, is necessary to take into account the voltage level at the end of the line, or at the PV plant location (which is called wide-area-control, with remotely measure nodes) [2].

Line Voltage Regulators (LVR), also known as Step Voltage Regulators (SVR) or Booster Transformers, are autotransformers used to stabilize voltage in long and heavily loaded feeders [2], which typically have a $\pm 10 \%$ voltage regulation capability [3]. In a PV integration context, they can be placed in the middle of the line to prevent voltage from overtaking the superior limit when 
voltage rise occurs. They are connected in series on the distribution line, and voltage is controlled based on the current passing through the device [4]. Figure 1 shows a voltage profile with and without the installation of a LVR.

Other commonly used strategy is reactive power control or VAR-control. Provision of reactive power through fastacting power electronic devices, such as Static VAR Compensators (SVC), Static Synchronous Compensators (STATCOMs) or other equipment such capacitor banks.

Grid reinforcement is used to improve voltage profiles by increasing the cross-sectional area of the feeder, reducing its impedance [1]. As mentioned before, this should be considered as a last resort, since it is the most expensive and complex. However, to confirm this assumption, a cost-benefit analysis should be performed among all the possible alternatives.

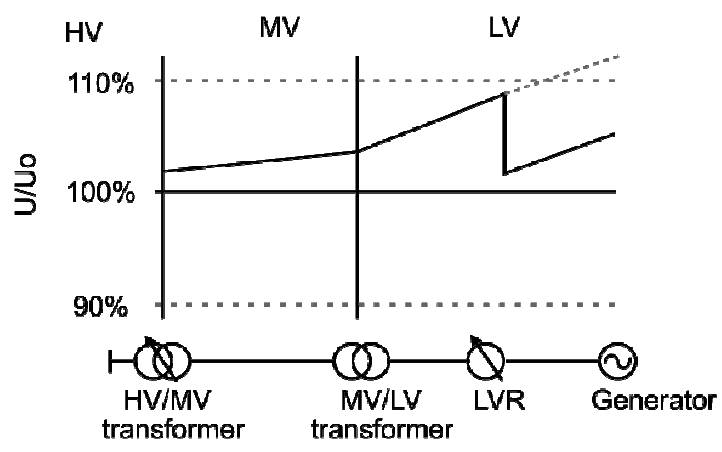

Fig. 1. Voltage profile with and without a LVR.

Network reconfiguration can be seen as a kind of grid reinforcement, although it does not necessarily imply changes in cables. An interesting option to study in the future is the possibility of automatic reconfiguration of the grid according to the conditions of the system [2].

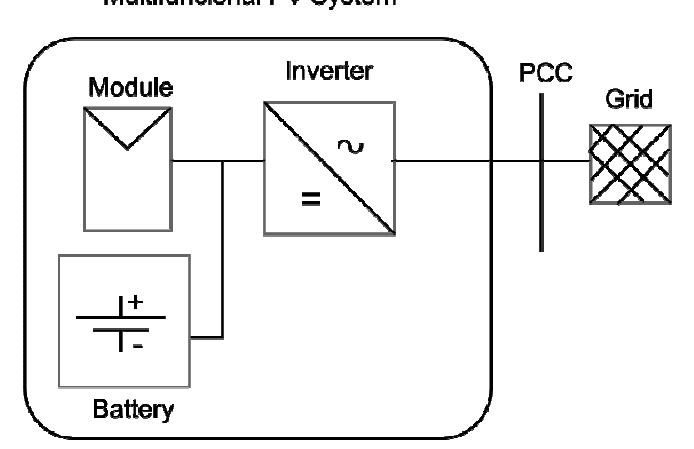

Fig. 2. Technical solutions that may be provided by prosumers [2].

An important matter that needs to be explained for the sake of a clear understanding of the subject is the
The main idea is operating LV networks in a similar method as high voltage grids. Closed-loop operation increases the short-circuit power of interconnection points in the grid, which increases the strength of the grid, reducing the effect of dispersed power feed-in on voltage (and thus, the severity of voltage rise) [2].

Another possibility to mitigate voltage fluctuation issues is the use of large scale electricity storage units along the distribution systems to manage active power flows. Depending on the type of storage technology, they can contribute to different control strategies.

\section{Solutions provided by PV Systems}

The prosumer concept is used to define those actors of the electricity system who actively interact with the electricity system: they consume but also produce electricity, can vary their demand patterns and can provide ancillary services to the network. In this sense, a PV system owner may be considered as a prosumer. Prosumers can help to mitigate several kinds of problems through a series of strategies including active power curtailment and reactive power compensations of PV systems, increasing selfconsumption and using storage devices or even shifting their demand to high solar production hours, through load management strategies [2].

In Figure 2, some of the technical services that smart inverters can provide to the electrical system are depicted. It is worth mentioning that for the provision of some of these services, storage systems are required. From all of the features that smart inverters and PV system in general have, reactive power compensation and active power management are the most interesting which can help with the integration of PV systems, contribute to grid stability and provide system-wide cost and performance efficiencies [5].

\section{Technical Services}

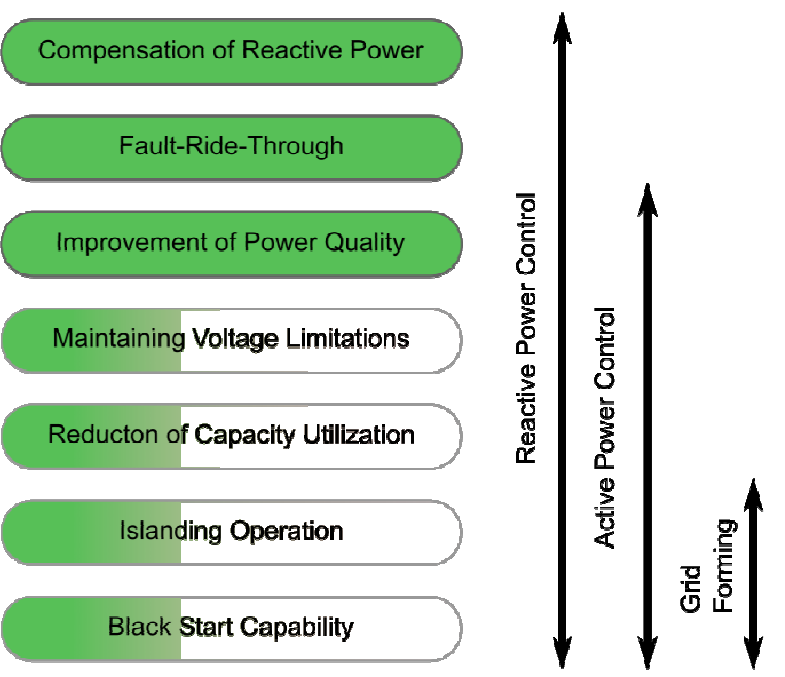

operational window of the inverters. Figure 3 shows the P$\mathrm{Q}$ diagram (active power in the vertical axis, reactive 
power in the horizontal axis) of a PV inverter. The semicircumference's radio is the rated apparent power of the inverter (S). At any point on this semi-circumference, the $\mathrm{PV}$ inverter will be operating at its maximum apparent power.

PV inverters are traditionally designed to operate with unity power factors i.e. operational points along the vertical axis, where the maximum power output corresponds to the intersection with the semicircumference. In order to use reactive power capabilities of smart inverters, there are two main options: limiting the amount of active power delivered (Figure 3, a), or oversizing the inverter (Figure 3, b). Obviously, the first of these options implies a reduction in the PV production and therefore, it would lead to reduced earnings for the PV system owner.

a)

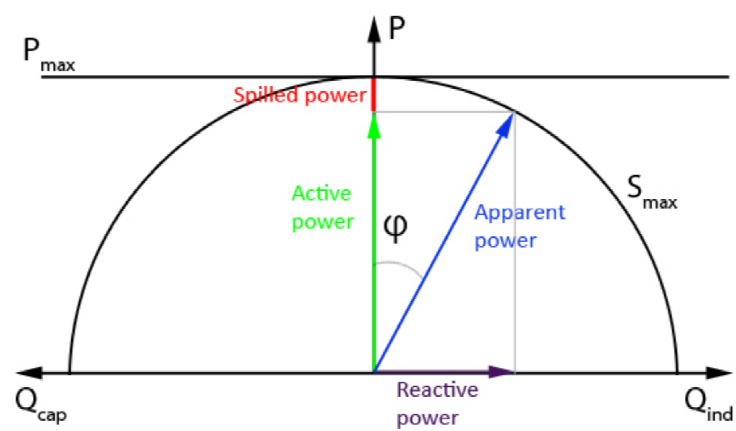

b)

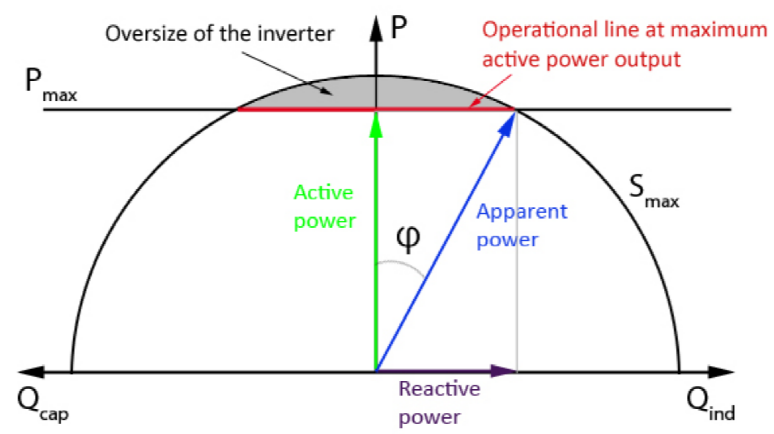

Fig 3. Operational window of PV inverters. a) Without oversizing inverter and b) oversizing it.

On the other hand, oversizing the PV inverter allows having reactive power compensation capabilities, while delivering full power output from its PV field. Of course, oversizing the inverter has an extra cost that needs to be taken into account, although the differences in prices are not large enough to become an obstacle in the decision.

\section{Active power curtailment}

The simplest mitigation method provided by PV inverters is the active power reduction or curtailment [2]. This strategy can be implemented in different ways, such as fixing a maximum point to active power (e.g. $70 \%$ of the rated power), or basing the reduction on a reference signal, as the voltage at the PCC. Figure 4 shows an operational curve that could be implemented in $\mathrm{PV}$ inverters using the later method. In this case, two operational points are set and if the voltage at the PCC increases beyond the specified limits, the inverters adjusts operation of the maximum power point tracking (MPPT) to reduce output power [6].

The high $\mathrm{R} / \mathrm{X}$ ratios in LV networks makes this measure quite effective in voltage reduction. However, active power curtailment also suppose an effective power spilling, and the PV generation will be less than it could be for the available solar irradiance [6]. This affects the overall economic viability of the PV system. While from the perspective of the DSO this measure has clear advantages, this strategy has a cost for PV system owners, hence this is a point of contention that needs to be fixed for a successful application [5].
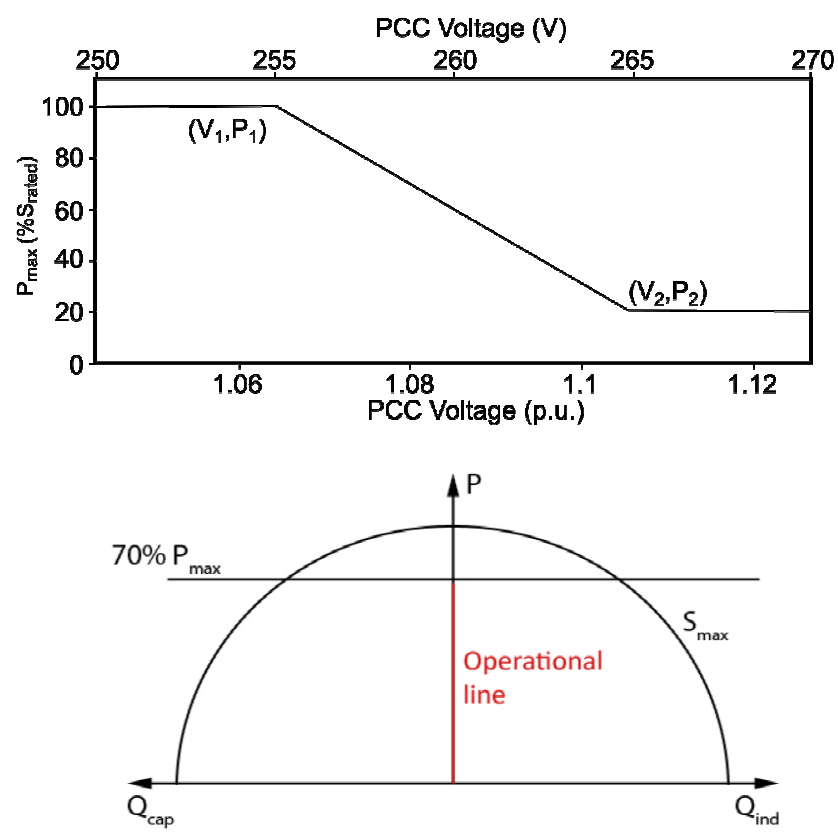

Fig. 4. Active power curtailment control and operational window. Source: [6].

\section{Reactive power compensation}

Reactive power compensation of smart inverters is an interesting option to manage network voltages through reactive power injections or absorptions [6]. There are two different types of reactive power support provided by PV inverters: dynamic provision and static provision.

Dynamic reactive power support is used to provide stability to the grid when some events like short-term voltage sags or peaks occur. Hence, inverters use dynamic reactive power provision for their "riding-through" capabilities.

Static reactive power support is used to maintain voltage levels within an acceptable range. The idea of using reactive power for maintaining voltage limitations derives from traditional power system operation, where reactive power is necessary to support the overall voltage stability in the grid [2].

However, the effectiveness of the measure will depend on the $\mathrm{R} / \mathrm{X}$ ratio of the grid. As in $\mathrm{LV}$ networks, higher $\mathrm{R} / \mathrm{X}$ 
ratios are found, reactive power compensation will be less effective than in higher voltage grids. Despite this fact, the purpose of providing reactive power compensation in $\mathrm{LV}$ networks is to produce additional power flows of reactive power which will decrease the voltage raises caused by distributed generation (DG) [2].

Current PV inverters usually can implement static reactive power compensation by means of four different control strategies: (i) fixed $\mathrm{Q}$, (ii) fixed $\cos \phi$, (iii) $\cos \phi(\mathrm{P})$ and (iv) Q (V). However, it is expected that more sophisticated control methods will appear, as proposed in [7].

\section{A. Fixed $Q$}

With the fixed Q method (Figure 5, a), the PV inverter delivers or absorbs the same amount of reactive power regardless other variables in the system. This method requires information of load power and PV power profiles in order to set an adequate Q point [7]. Since this strategy does not take into account other variables in the system, such as voltage, the PV inverters will provide reactive power compensation even when it is not required.
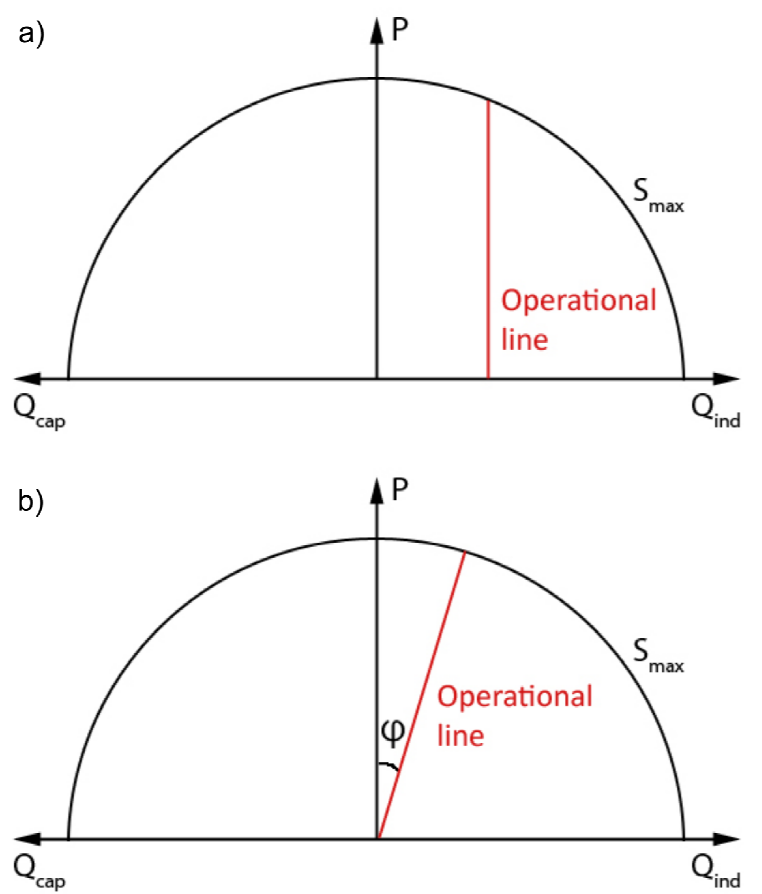

Fig. 5. Operational windows for (a) Fixed Q control and (b) Fixed $\cos \phi$ control.

\section{A. Fixed $\cos \phi$}

With fixed power factor $(\cos \phi)$, the generated reactive power is proportional to the active power (Figure 5, b). The PV inverter is forced to work at a determined $\cos \phi$, again without taking into consideration system variables. For that reason, the inverter will be providing reactive power compensation although it may be not needed, as it happens with the fixed Q method.

\section{B. $\cos \phi(P)$}

The $\cos \phi(\mathrm{P})$ strategy is based on the measure of the active power output of the PV inverter. The concept is to vary the power factor (providing reactive power compensation) when power output reaches certain value e.g. $50 \%$ of rated power. Figure 6 depicts a characteristic curve of this control strategy.
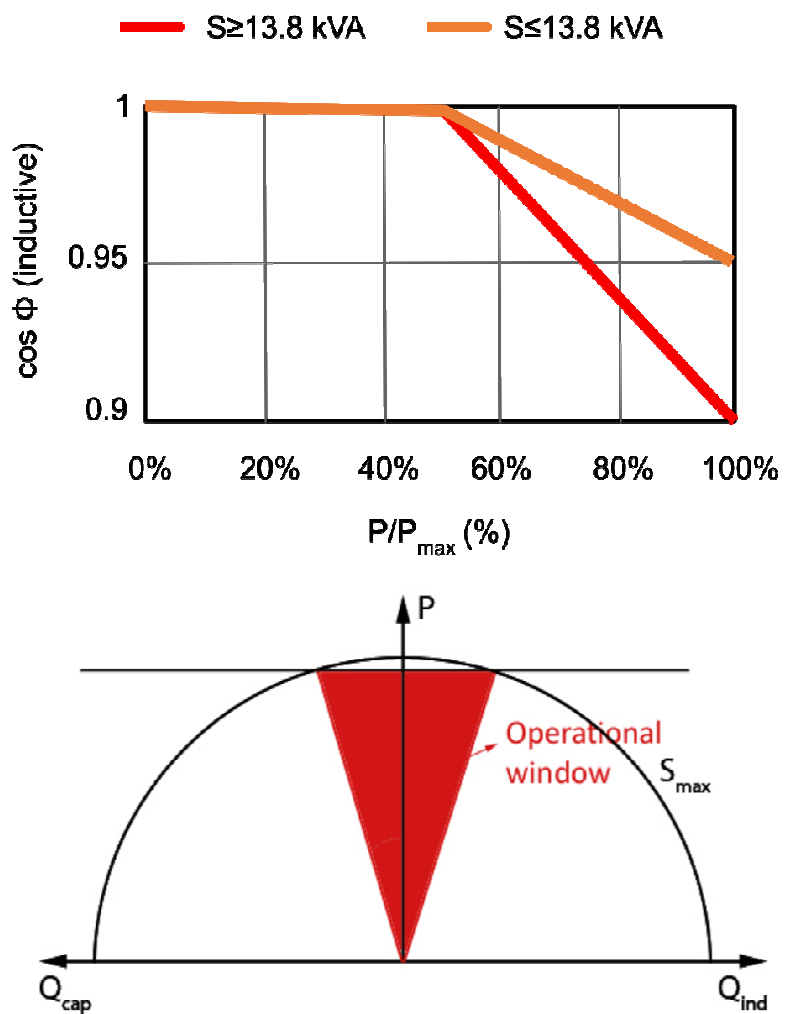

Fig. 6. Characteristic curve and operational window of $\cos \phi(\mathrm{P})$ control.

C. $Q(V)$

In the $\mathrm{Q}(\mathrm{V})$ method, also referred to as volt-var function, the PV inverter will provide an amount of reactive power as a function of the voltage at its point of interconnection, accordingly to the setting points programmed in the inverter [13]. Figure 7 shows an example of a characteristic curve that may be set in the inverter.

Following the depicted curve, the inverter will start to inject or absorb reactive energy (i.e. operate with capacitive or inductive power factors) when the registered voltage is outside the $\pm 4 \%$ range from the nominal voltage. If the voltage reaches the $\pm 7 \%$ limits, then all the available capacity for reactive power will be used.

Both strategies, $\cos \phi(\mathrm{P})$ and $\mathrm{Q}(\mathrm{V})$ methods, are local control functions, influenced by local variables of the inverter or the grid. However, if communication infrastructures are used, these strategies can be implemented in a global control fashion, optimizing the total assets of the network and providing better results of voltage regulation (some authors called it centralized control [6]). 

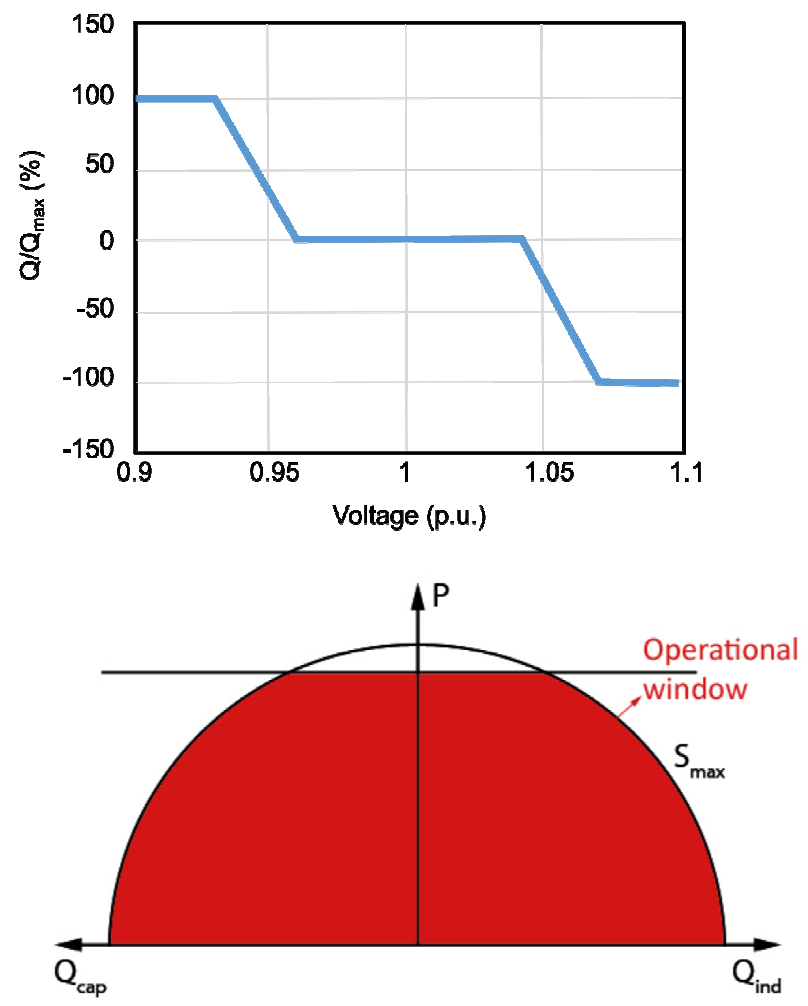

Fig. 7. Characteristic curve and operational window of $Q(V)$ control.

\section{Load management strategies and self-consumption}

Another solution that prosumers can provide to the distribution system is trying to match their demand patterns with the PV production. This can be made both by shifting their non-critical demands to the hours with highest PV generation or by increasing self-consumption instead of delivering all produced power to the network. It is obvious that if less power is introduced into the electric grid, the occurrence of voltage rise will be reduced.

\section{E. Storage systems}

Storage systems are another way of somehow increasing self-consumption. The idea is to store the surplus of PV power instead of injecting it into the network, thus reducing the probability of voltage rise to occur. Furthermore, using energy management systems (EMS), the demand profile at the point of common coupling could be smoothed out by levelling load and generation [2]. However, different storage technologies provide different services, and along with the storage size selected, they will determine for what purpose could be used (peak shaving, increase of self-consumption, ramp rate control...).

Nevertheless, some novel concepts of storage technologies known as Hybrid Energy Storage Systems (HESS) are being developed. They are usually form by two or more complementary technologies which allows for broadening the operational range of storage systems [8].

\section{Importance of regulatory frameworks and grid codes}

In general, electricity grids must meet a series of strict requirements regarding the quality of the electricity delivered, in order to ensure a safe and effective operation by the end-user loads and infrastructures. This is particularly true in low voltage distribution grids, where the majority of customers are connected to draw electricity from the network. This set of operational conditions are usually referred to as power quality requirements, and they are regulated in standards, laws, grid codes or even by utility companies in some countries [4].

The maintenance of these power quality requirements is one of the reasons why the interconnection of DG at LV is so problematic. LV distributions networks are inherently difficult to operate, since they have a stochastic behaviour that depends on multiple uncontrollable factors, such as the use that end-users make of their loads. Traditionally, in a radially operated network, these quality parameters are adjusted by the DSO, at the substation, using tap changers in the transformers or capacitor banks [1]. Nevertheless, when the grid starts to experience multidirectional power flows due to higher PV penetration rates, the complexity of operation increases.

This fact evidences the necessity of having robust frameworks and grid codes that regulate the interconnection of distributed energy sources to LV networks while maintaining the quality standards and the reliability of the electric system.

The IEA, within the Task 14 of its Photovoltaic Power Systems (PV-PS) program, has identified and studied the regulatory and technical $\mathrm{PV}$ integration issues in distribution grids of interconnected electric power systems. According to the IEA PV-PS Task 14, the electric system of every country that is going to increase the PV share to the total electricity mix, will face three different stages (which are depicted in Figure 8), each of them with different issues to be addressed [2]:

a) Stage 1. Low/medium PV penetration in a few distribution grids, where local consumption is higher than local generation

b) Stage 2. High PV penetration in a few distribution grids, where local generation exceeds local consumption

c) Stage 3. High PV penetration in many distribution grids, where PV will become a major electricity source

In order to achieve a cost-effective and reliable transition from one stage to another, advanced technology such as smart inverters, as well as robust regulatory frameworks are needed to be adopted as soon as possible. The late implementation of grid codes will result in high integration costs due to the necessary adaptation of existing PV systems [2]. 
Germany has recently developed a grid code (VDE-AR-N 4105) that regulates the interconnection of DG in $L V$ networks. The main innovative measure included is the requirement of active and reactive power compensation by distributed generators [9]. In the USA, a revision for their IEEE 1547 standard is being prepared, that will provide more flexibility to the interconnection of PV generators allowing advanced functionalities of the inverters to provide voltage control at the distribution level [4]. Other countries with LV interconnection grid codes, such as
Italy, Belgium, Japan or Australia, do not have any active or reactive power regulations. The European Network of Transmission System Operators for Electricity (ENTSOE) is preparing a new grid code, which, once approved, will rule the requirements for grid connections for any generators, at all voltage levels. This grid code will not make any statement of provision the regulation services, but it will require the capability to provide them [4].

\section{DSO/TSO Observations}

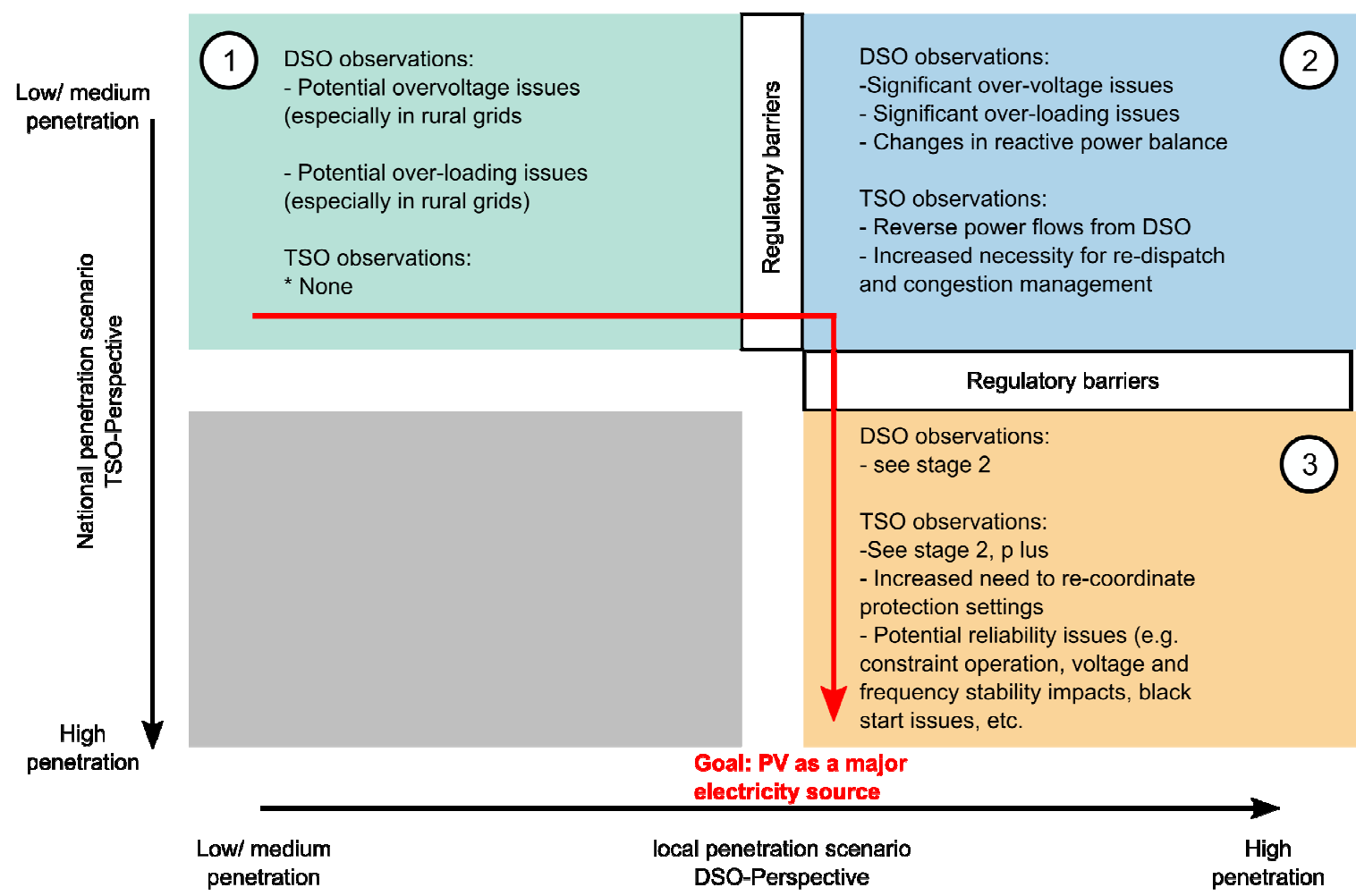

Fig. 8. Stages in the transition from uni- to multi-directional distribution systems. Source: [2].

Taking into account the future perspectives of PV penetration, it seems mandatory that policy makers in Spain and Canary Islands start to develop a regulatory framework which allows the use of advanced functionalities of PV inverters (and any DG), facilitating the deployment of PV system and enabling a distributed regulation infrastructure at the distribution level.

\section{Conclusions}

The increasing penetration of PV systems into LV networks is leading to power quality issues and integration problems. Within Spain, it should be expected that the deployment of PV systems in Canary Islands will be larger than in other locations. High irradiation levels and high cost of conventional electricity productions will drive this deployment. However, electric systems in small islands are relatively weaker than continental systems, and therefore, the probability of problems to arise is likely to be higher. Hence, special attention should be paid to the PV integration issues in Canary Islands.
The most problematic and limiting factor in the deployment of PV systems is the voltage rise, but problems related to power fluctuations, frequency or harmonics (among others) may also occur with high PV penetration rates. In this paper, both the DSO and PV system owner have addressed most of the problems related with PV integration in LV system, as well as the possible solutions that may be adopted.

Among the solutions available for the DSO (which are mainly derived from the experience in the voltage control of medium and high voltage networks), the implementation of LVR seems very interesting, since this equipment provides solutions for both under and overvoltage situations.

Regarding the solutions that PV system owners may provide, the use of advanced inverters with capabilities of active power curtailment and reactive power compensation is very attractive. The higher $\mathrm{R} / \mathrm{X}$ ratios of $\mathrm{LV}$ lines make that active power flows have more influence over voltage than reactive power flows, making the first measure more efficient than the later. However, the effect that reactive power compensation has over the 
voltage cannot be neglected. In fact, this procedure is a promising way to enable a distributed regulation system through the LV network with already deployed resources.

Finally, regarding the real case studied, there are several issues that still needs to be clarified, like the cause of the pronounced voltage unbalance or the influence of the capacitor bank over the problem. Additionally, it would be interesting to study the effect that a PV inverter, which performs reactive power compensation individually in each of the phases, would have over both the voltage rise and voltage unbalance issues.

\section{Acknowledgement}

This work has been supported by the Fundación Cajacanarias (Project 2016TUR17).

\section{References}

[1] M. Mejbaul Haque and P. Wolfs, "A review of high PV penetrations in LV distribution networks : Present status , impacts and mitigation measures," Renew. Sustain. Energy Rev., vol. 62, pp. 1195-1208, 2016.

[2] T. Stetz, M. Rekinger, and I. T. Theologitis, "Transition from Uni-Directional to Bi-Directional Distribution Grids," 2014, http://iea-

pvps.org/fileadmin/dam/intranet/ExCo/Transition from uni dire ctional_to_bi_directional_distribution_grids_REPORT_PVPS_T 1403 2014.pdf, consulted in January 2018.

[3] Y. P. Agalgaonkar, B. C. Pal, and R. A. Jabr, "Distribution voltage control considering the impact of PV generation on tap changers and autonomous regulators," IEEE Trans. Power Syst., vol. 29, no. 1, pp. 182-192, 2014.

[4] T. Ehara, "Overcoming PV grid issues in the urban areas," 2009. http://www.iea-pvps-task10.org/IMG/pdf/rep10_06.pdf, consulted in January 2018.

[5] National Renewable Energy Laboratory (NREL), "Advanced Inverter Functions To Support High Levels of Distributed Solar Policy and Regulatory the Need for Advanced," 2014. https://www.nrel.gov/docs/fy15osti/62612.pdf, consulted in January 2018.

[6] K. Turitsyn, P. Sulc, and M. Chertkov, "Local Control of Reactive Power by Distributed Photovoltaic Generators," pp. 7984.

[7] E. Demirok, P. C. González, K. H. B. Frederiksen, D. Sera, P. Rodriguez, and R. Teodorescu, "Local reactive power control methods for overvoltage prevention of distributed solar inverters in low-voltage grids," IEEE J. Photovoltaics, vol. 1, $\mathrm{n}^{\circ}$. 2, pp. 174-182, 2011.

[8] A. Etxeberria, I. Vechiu, H. Camblong, J. M. Vinassa, and H. Camblong, "Hybrid Energy Storage Systems for renewable Energy Sources Integration in microgrids: A review," 2010 Conf. Proc. IPEC, pp. 532-537, 2010.

[9] L. Schwartfeger and D. Santos-Martin, "Review of Distributed Generation Interconnection Standards," IEEE Electr. Power Conf., vol. 172, p. 13, 2014. 